\title{
The Quality of Cryopreserved Sperm Collected from Feline Caudal Epididymides Using Seminal Plasma
}

\author{
Mari TOYONAGA ${ }^{1,2) *}$ and Toshihiko TSUTSUI ${ }^{1,2}$ \\ 1) Department of Reproduction, Nippon Veterinary and Life Science University, 1-7-1 Kyonan-cho, Musashino-shi, Tokyo 180-8602, Japan \\ 2) International Institute of Small Animal Medicine, Bio Plus, AHB Inc., 2-7-2 Sumiyoshi, Koutou-ku, Tokyo 135-0002, Japan
}

(Received 12 April 2011/Accepted 14 May 2012/Published online in J-STAGE 28 May 2012)

ABSTRACT. It is thought that differences in conception rate between feline epididymal sperm and ejaculate sperm occur because, unlike ejaculated sperm, caudal epididymal sperm have not been sensitized with seminal plasma (SP). In this study, we investigated whether collection of feline epididymal sperm with SP influences sperm qualities after freezing-thawing. Sperm were sensitized with SP for 10 min at room temperature. As a result, the motility of caudal epididymal sperm sensitized with SP immediately after collection was significantly lower than that of ejaculate sperm, and no difference was noted in sperm qualities after freezing-thawing. This shows that the qualities of caudal epididymal sperm cannot be improved to a level higher than those of ejaculate sperm by sensitization with SP.

KEY WORDS: epididymal sperm, feline, seminal plasma.

doi: 10.1292/jvms.11-0175; J. Vet. Med. Sci. 74(10): 1349-1353, 2012

Since many wild feline species are on the verge of extinction, many researchers are investigating gamete preservation techniques $[1,2,11,13,14,16]$. We also previously reported on the acquisition of newborns by artificial insemination of frozen semen collected from the feline caudal epididymis using egg yolk Tris-fructose citrate (EYT-FC), but the conception rate was low [14]. However, regarding the qualities of semen used, sperm motility and viability were similar to those of feline frozen ejaculated semen used in artificial insemination as previously reported [15]. The lack of sensitization of epididymal sperm with seminal plasma (SP) was considered to be the cause of this difference. To investigate this difference, we observed glycoprotein attachment in epididymal sperm not sensitized with SP and sensitized ejaculated sperm using 8 types of lectin [12]. However, no difference was noted in lectin binding between feline epididymal and ejaculated sperm. The effects of SP on sperm functions have been reported to be positive or negative in various animal species [3, 4, 6-8, 11].

Kawakami et al. [5] investigated protein attachment on the surfaces of canine caudal epididymal and ejaculated sperm. They observed that PHA-E lectin did not bind to caudal epididymal sperm but bound to ejaculated sperm and suggested that a PHA-E lectin-binding glycoprotein, $\mathrm{N}$-acetyl-D-galactosamine, is a decapacitation factor. Hori et al. [4] collected canine caudal epididymal sperm using SP and found that sperm qualities after freezing-thawing were apparently superior to those of ejaculated semen and that the conception rate after intrauterine artificial insemination was

\footnotetext{
*Correspondence to: Toyonaga, M., International Institute of Small Animal Medicine, Bio Plus, AHB Inc., 2-7-2 Sumiyoshi, Koutou-ku, Tokyo 135-0002, Japan. e-mail: marimaru@sd5.so-net.ne.jp

(C)2012 The Japanese Society of Veterinary Science
}

apparently higher. However, they did not clarify the reason why epididymal sperm collected with SP was superior to ejaculated sperm.

In this study, we investigated whether collection of feline epididymal sperm with SP improves sperm qualities after freezing-thawing.

Epididymal sperm from 5 male mixed domestic cats of known age that had been brought to an animal hospital for castration were used in this study. The cats ranged in age from 2.0 to 7.0 years old $(3.2 \pm 0.6$ (SE) years old, mean $\pm \mathrm{SE})$ and weighed 4.3 to $6.8 \mathrm{~kg}(5.3 \pm 0.5 \mathrm{~kg})$. Ejaculated sperm used in the experiment were collected from 7 cats aged 6.2-6.7 years $(6.4 \pm 0.1$ years $)$ and weighing 3.8 to 4.7 $\mathrm{kg}(4.1 \pm 0.2 \mathrm{~kg})$. They had been bred in our laboratory and exhibited normal breeding behavior and fertility. These cats were housed in $60 \times 90 \times 120$ (height)-cm stainless steel cages in an animal room maintained at a temperature of $22 \pm$ $2^{\circ} \mathrm{C}$ under a 12-hr lighting cycle, fed dry cat food and given free access to drinking water. The study was conducted in conformity with the animal study guidelines of Nippon Veterinary and Life Science University.

Tomcats were anesthetized using medetomidine hydrochloride (Domitol 0.05 mg/kg, i.m.; Nippon Zenyaku Kogyo Co., Ltd., Tokyo, Japan) and ketamine hydrochloride (Ketalar 5 mg/kg; Sankyo Co., Ltd., Tokyo, Japan). Immediately after castration, the cats were awakened by treatment with atipamezole hydrochloride (Antisedan, $0.025 \mathrm{mg} / \mathrm{kg}$, i.m.; Nippon Zenyaku Kogyo Co., Ltd.). After ligating the vas deferens and pampiniform venous plexus, the excised bilateral testis and epididymis of each cat were stored in sterile physiological saline to prevent drying. The caudal epididymis was incised at several sites using surgical scissors, and caudal epididymal sperm were collected into 200 $\mu l$ of recovery solution in a small pointed-bottom tube. For the recovery solution, SP was used on one side (SP group) and EYT-FC [9] on the other (EYT-FC group). Ejaculated 


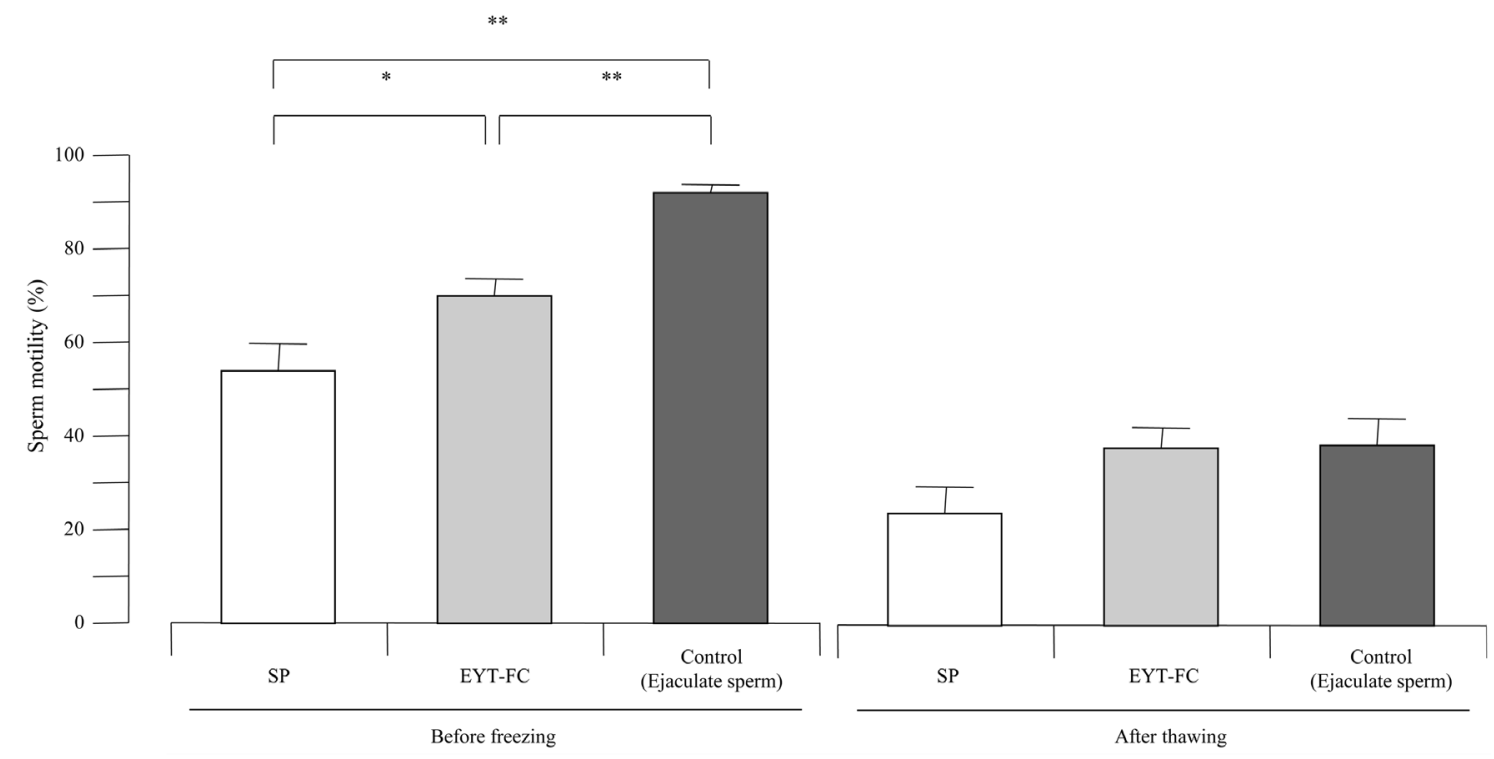

Fig. 1. Sperm motility of before freezing and frozen-thawed caudal epididymal cat sperm recovered using SP or EYT-FC. Values are means \pm SE of 5 cats. One-way ANOVA $\left({ }^{*} P<0.05 ; * * P<0.01\right)$.

sperm were used as the control group. SP was collected by low-temperature centrifugation $(3,000 \mathrm{rpm} \times 10 \mathrm{~min})$ immediately after semen collection and stored at $-40^{\circ} \mathrm{C}$ until the experiment. SP collected from 7 male cats was mixed and used. Sperm were sensitized with recovery solution for 10 min at room temperature. Sperm suspensions that had been collected were centrifuged at $1,500 \mathrm{rpm} \times$ for $5 \mathrm{~min}$, and after removing supernatants, $200 \mu l$ of EYT-FC was added to the sperm. Ejaculated sperm (control group) were collected using a feline artificial vagina and an estrous female as a prop and for sexual stimulation, respectively. The quality of sperm from these three groups, i.e., sperm motility, sperm viability, percentage of abnormal sperm and percentage of immature sperm, was evaluated as previously reported [9].

Sperm was cryopreserved according to the method previously reported [13]. Semen was subjected to the 1 st dilution at $20^{\circ} \mathrm{C}$ to adjust the sperm count to $1 \times 10^{8} / \mathrm{m} l$ and was then kept in a water bath at $4^{\circ} \mathrm{C}$ for $1 \mathrm{hr}$ for a $1 \mathrm{st}$ cooling. After the 1 st cooling, a secondary extender with $14 \%$ glycerol and $2.0 \%$ Orvus Es Paste (OEP, Nova Chemical Sales Inc., Scituate, MA, U.S.A.) was added by dripping at $4^{\circ} \mathrm{C}$, taking approximately $10 \mathrm{~min}$ with stirring. Semen was diluted with an equal volume of the extender, giving a final sperm concentration of $0.5 \times 10^{8} / \mathrm{m} l$ and final glycerol and OEP concentrations of 7 and $1 \%$, respectively. After the 2 nd dilution, semen was put into $250 \mu l$ straws, and equilibrated with glycerol for $1 \mathrm{hr}$. For freezing, liquid nitrogen $\left(\mathrm{LN}_{2}\right)$ was poured into a styrene foam box $[30.5 \times 23 \times 16.5$ (height) $\mathrm{cm}$ ] to a level of $5 \mathrm{~cm}$, and the straws were maintained horizontally at a height of $7 \mathrm{~cm}$ from the surface for $10 \mathrm{~min}$ in $\mathrm{LN}_{2}$ vapor. The straws were then plunged into the $\mathrm{LN}_{2}$. Frozen straws were stored for more than 1 week, and thawed by immersing them in $37^{\circ} \mathrm{C}$ water for $30 \mathrm{sec}$. The sperm quality test was performed immediately after thawing as previously reported [9].

Epididymal sperm were collected from 5 male cats, ejaculated sperm were collected one time from each of the 7 male cats, and the mean values of these data were compared. This experiment was conducted just once. All data are shown as means \pm SE. The significance of differences in sperm quality among the groups was analyzed by one-way ANOVA and the Tukey-Kramer test. A $P$-value of less than 0.05 was regarded as significant.

Mean sperm motilities immediately after collection in the SP, EYT-FC and control groups were 54.0 $\pm 5.7,70.0 \pm 3.5$ and $92.1 \pm 1.6 \%$, respectively, showing that sperm motility was significantly lower in the SP group than in the EYT-FC and control groups and was significantly lower in the EYTFC group than in the control group (Fig. 1). Mean sperm motilities after freezing-thawing were $24.0 \pm 5.7,38.0 \pm 4.2$ and $38.6 \pm 5.5 \%$, respectively, showing no significant differences (Fig. 1). Mean sperm viabilities immediately after collection revealed that viability was significantly lower in the SP group than in the EYT-FC and control groups and was significantly lower in the EYT-FC group than in the control group (Fig. 2). Mean sperm viabilities after freezing-thawing showed no significant differences (Fig. 2). Mean percentages of abnormal sperm found either before freezing and or after freezing-thawing showed no significant differences among the 3 groups (Fig. 3). Sperm abnormalities, mainly coiling and bending, were frequently observed in the tail. Mean incidences of immature sperm immediately after collection and those after freezing-thawing showed no significant differences among the groups (Fig. 4).

The motility and viability of SP-sensitized feline caudal epididymal sperm immediately after collection were signifi- 


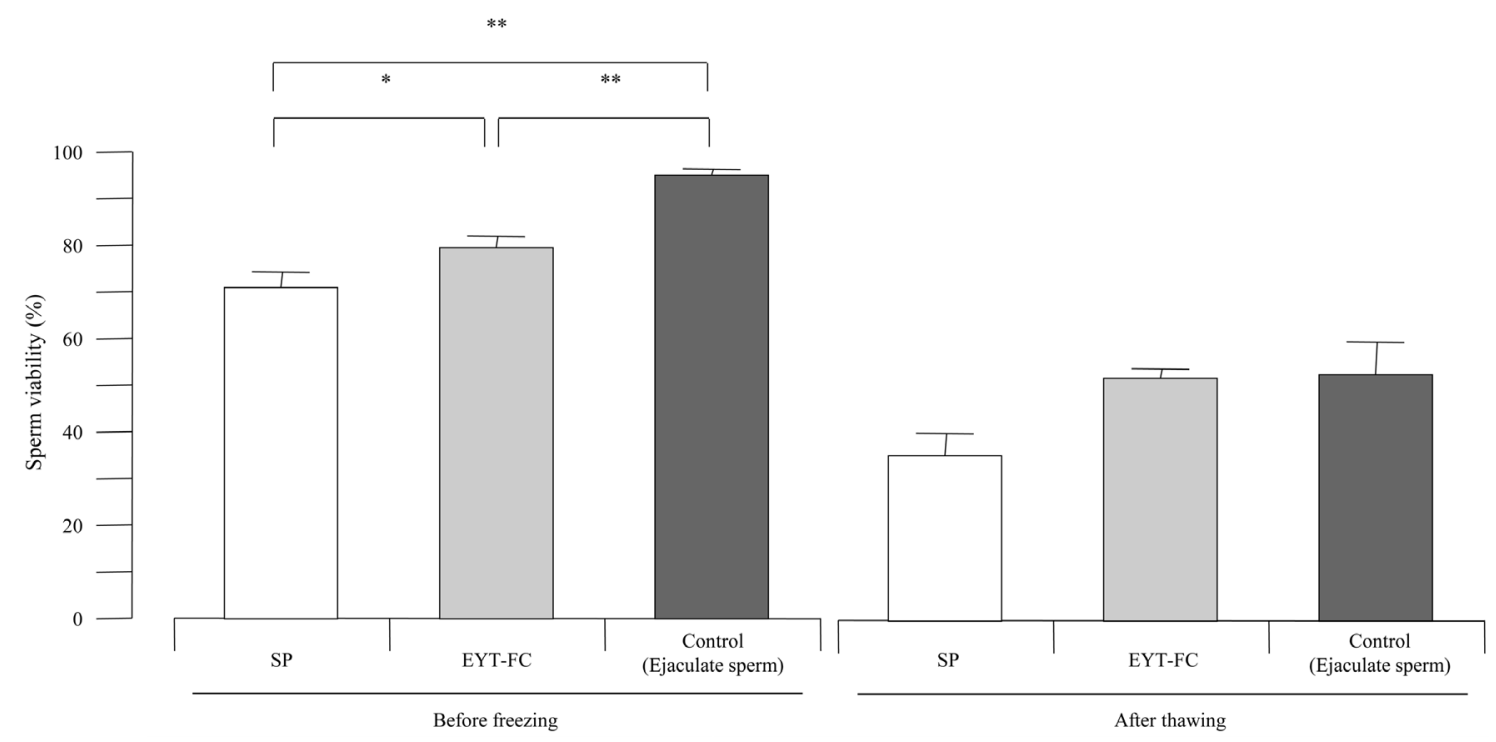

Fig. 2. Sperm viability of before freezing and frozen-thawed caudal epididymal cat sperm recovered using SP or EYT-FC. Values are means $\pm \mathrm{SE}$ of 5 cats. One-way ANOVA $(* P<0.05 ; * * P<0.01)$.

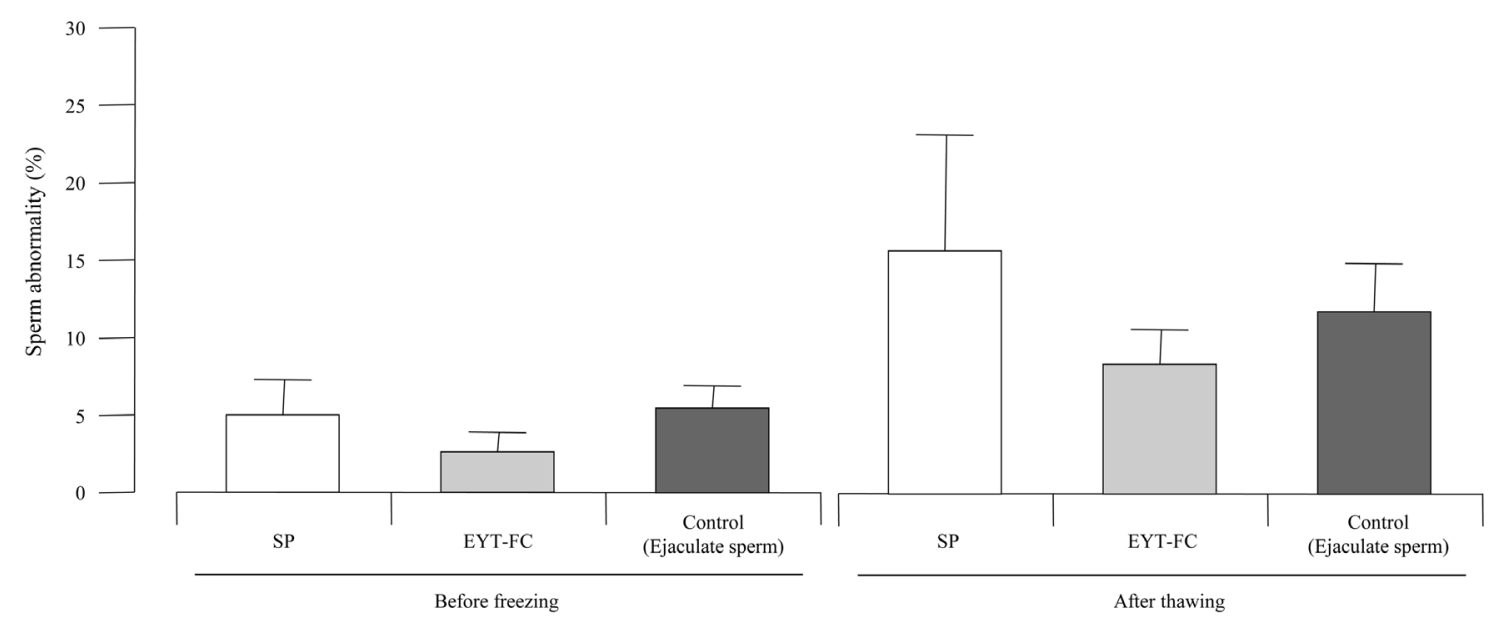

Fig. 3. Sperm abnormality of before freezing and frozen-thawed caudal epididymal cat sperm recovered using SP or EYT-FC. Values are means \pm SE of 5 cats.

cantly lower than those in the EYT-FC and control groups. We considered that SP-sensitized epididymal sperm were mostly equivalent to ejaculated sperm, but the qualities of sperm collected with SP were significantly lower than those of caudal epididymal sperm collected with EYT-FC. Thuwanut et al. [11] cultured feline frozen-thawed epididymal sperm with SP. They observed no significant differences in sperm motility either with or without SP treatment, showing that addition of SP to frozen-thawed epididymal sperm was useless. Although they did not investigate the direct effect of SP on freezing because sperm was cultured with SP after freezing-thawing, the uselessness of SP for frozenthawed epididymal sperm was consistent with the findings of the present study. Moreover, SP from several animals was mixed and then frozen until use in both their and our studies. However, the SP used by Hori et al. [4] to sensitize epididymal sperm from dogs was not a mixture, and when this cryopreserved SP was used to collect caudal sperm, sperm motility after thawing was improved, showing no influence of freezing. In cats, mixing and freezing of SP from several animals may have caused the lack of a significant effect on sperm quality, but comparison with dogs was impossible because the feline SP volume is small and the use of fresh SP collected from a single animal in experiments is difficult. Although there has been no report in which sperm qualities were compared between ejaculated sperm collected using the artificial vagina method and caudal epididymal sperm, Tebet et al. [10] compared sperm qualities between semen 


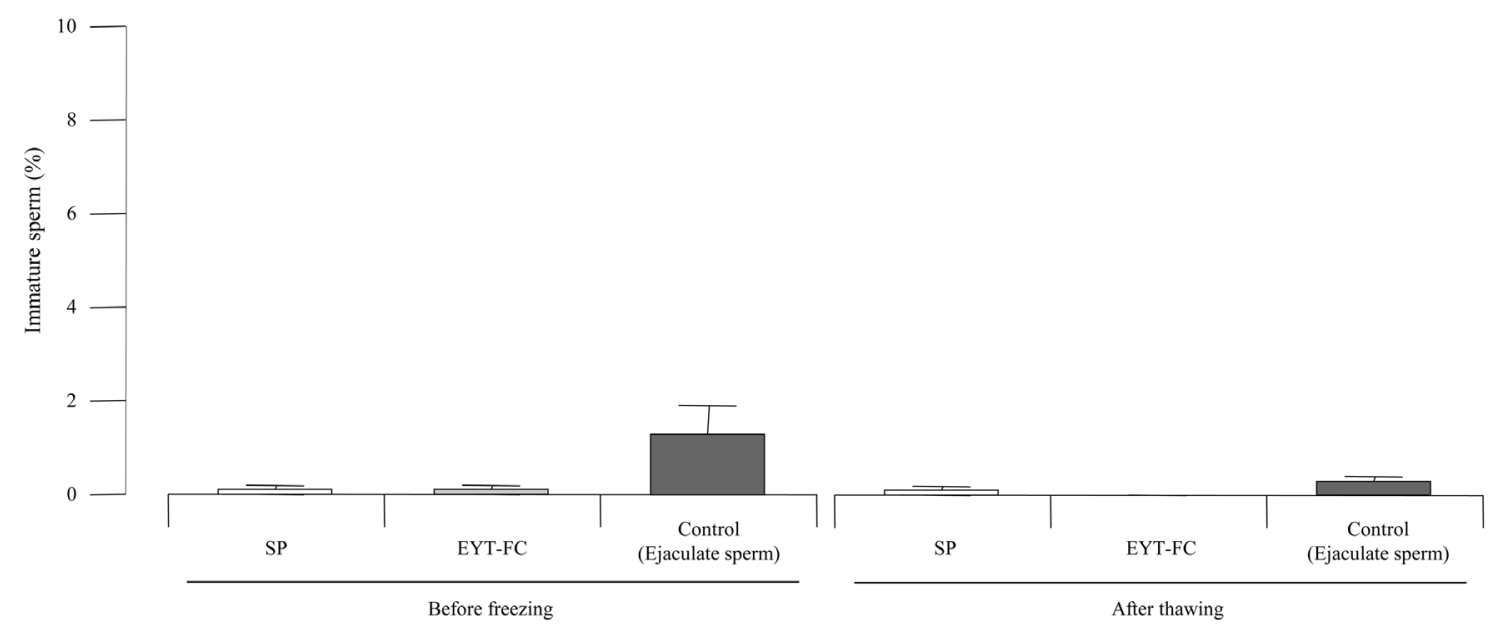

Fig. 4. Immature sperm from before freezing and frozen-thawed caudal epididymal cat sperm recovered using SP or EYT-FC. Values are means \pm SE of 5 cats.

collected by electroejaculation and caudal epididymal sperm collected by flushing the seminal duct and observed no significant difference between groups. In the mincing method employed by us to collect sperm, the sperm recovery solution contained epididymal tissue and blood. These may have influenced sperm and reduced their motility.

In other animal species, improvement in sperm qualities or fertility by SP has been frequently reported [3, 4, 6, 7]. For example, in Iberian red deer [6], no difference due to the presence or absence of SP was noted in epididymal sperm motility before freezing, but motility was significantly higher when sperm were treated with SP after freezing-thawing. The same phenomenon has been reported $[4,7]$ in dogs. In horses [3], it has been reported that the conception rate in artificial insemination was increased by addition of SP with either non-frozen or frozen-thawed sperm. In contrast, as an example of the negative action of SP sensitization, Rota et al. [8] reported that SP-treated canine epididymal sperm motility was significantly lower than that without SP treatment. However, they did not observe sperm quality after freezing-thawing. These studies reported that SP addition was useful because SP protected the sperm cell membrane and acrosomes. In the present study, we did not observe acrosomes, but the low SP-sensitized epididymal sperm motility suggested that the acrosome retention rates were low. Investigation of these factors and the conception rate using this semen is necessary.

No significant differences were noted in sperm qualities after freezing-thawing among the 3 groups, suggesting that sperm with marked viability survived the freezing process in ejaculated and epididymal sperm, and their proportions were similar in the 2 sperm groups.

SP sensitization of feline epididymal sperm collected by mincing the epididymis did not achieve qualities equivalent to those of ejaculated sperm, and sperm motility and viability were significantly lower than those of the other groups. In conclusion, SP sensitization with SP for $10 \mathrm{~min}$ at room temperature of feline epididymal sperm may be counterproductive for cryopreservation.

\section{REFERENCES}

1. Chatdarong, K., Thuwanut, P., Suksamai, P., Patanatiradaj, S. and Sangwornrachasup, A. 2009. Survival of frozen-thawed cat spermatozoa pre-cooled in the epididymides. Reprod. Domest. Anim. 44: 377-380. [Medline] [CrossRef]

2. Hay, M. A. and Goodrowe, K. L. 1993. Comparative cryopreservation and capacitation of spermatozoa from epididymides and vasa differentia of the domestic cat. J. Reprod. Fertil. Suppl. 47: 297-305. [Medline]

3. Heise, A., Kähn, W., Volkmann, D. H., Thompson, P. N. and Gerber, D. 2010. Influence of seminal plasma on fertility of fresh and frozen-thawed stallion epididymal spermatozoa. Anim. Reprod. Sci. 118: 48-53. [Medline] [CrossRef]

4. Hori, T., Hagiuda, K., Kawakami, E. and Tsutsui, T. 2005. Unilateral intrauterine insemination with prostatic fluid-sensitized frozen caudal epididymal sperm in beagle dogs. Theriogenology 63: 1573-1583. [Medline] [CrossRef]

5. Kawakami, E., Morita, Y., Hori, T. and Tsutsui, T. 2002. Lectinbinding characteristics and capacitation of canine epididymal spermatozoa. J. Vet. Med. Sci. 64: 543-549. [Medline] [CrossRef]

6. Martínez-Pastor, F., Anel, L., Guerra, C., Alvarez, M., Soler, A. J., Garde, J. J., Chamorro, C. and de Paz, P. 2006. Seminal plasma improves cryopreservation of Iberian red deer epididymal sperm. Theriogenology 66: 1847-1856. [Medline] [CrossRef]

7. Nöthling, J. O., Gerber, D., Colenbrander, B., Dijkstra, M., Bakker, T. and De Cramer, K. 2007. The effect of homologous prostatic fluid on motility and morphology of dog epididymal spermatozoa extended and frozen in Biladyl with Equex STM paste or Andromed. Theriogenology 67: 264-275. [Medline] [CrossRef]

8. Rota, A., Ström, B. and Linde-Forsberg, C. 1995. Effects of seminal plasma and three extenders on canine semen stored at $4^{\circ} \mathrm{C}$. Theriogenology 44: 885-900. [Medline] [CrossRef]

9. Tanaka, A., Kuwabara, S., Takagi, Y., Nakagawa, K., Fujimoto, Y., Murai, M. and Tsutsui, T. 2000. Effect of ejaculation intervals 
on semen quality in cats. J. Vet. Med. Sci. 62: 1157-1161. [Medline] [CrossRef]

10. Tebet, J. M., Martins, M. I. M., Chirinea, V. H., Souza, F. F., Campagnol, D. and Lopes, M. D. 2006. Cryopreservation effects on domestic cat epididymal versus electroejaculated spermatozoa. Theriogenology 66: 1629-1632. [Medline] [CrossRef]

11. Thuwanut, P., Chatdarong, K., Techakumphu, M. and Axnér, E. 2008. The effect of antioxidants on motility, viability, acrosome integrity and DNA integrity of frozen-thawed epididymal cat spermatozoa. Theriogenology 70: 233-240. [Medline] [CrossRef]

12. Toyonaga, M., Morita, M., Hori, T. and Tsutsui, T. 2011. Distribution of glycoproteins on feline testicular sperm, epididymal sperm and ejaculated sperm. J. Vet. Med. Sci. 73: 827-829. [Medline] [CrossRef]

13. Toyonaga, M., Sato, Y., Morita, M., Watanabe, M., Oba, H.,
Mizutani, T., Hori, T. and Tsutsui, T. 2010. The qualities of cryopreserved epididymal sperm collected from feline epididymides stored at low temperature. J. Vet. Med. Sci. 72: 777-780. [Medline] [CrossRef]

14. Tsutsui, T., Wada, M., Anzai, M. and Hori, T. 2003. Artificial insemination with frozen epididymal sperm in cats. J. Vet. Med. Sci. 65: 397-399. [Medline] [CrossRef]

15. Tsutsui, T., Mizutani, T., Matsubara, Y., Toyonaga, M., Oba, H. and Hori, T. 2011. Surgical intrauterine insemination with cat semen cryopreserved with Orvus ES paste or sodium lauryl sulfate. J. Vet. Med. Sci. 73: 259-262. [Medline] [CrossRef]

16. Tsutsui, T., Tanaka, A., Takagi, Y., Nakagawa, K., Fujimoto, Y., Murai, M., Anzai, M. and Hori, T. 2000. Unilateral intrauterine horn insemination of frozen semen in cats. J. Vet. Med. Sci. 62: 1247-1251. [Medline] [CrossRef] 